\title{
ESCALAS QUE EVALÚAN BARRERAS Y RECURSOS AMBIENTALES PARA BUSCAR AYUDA EN UNIVERSITARIOS QUE BEBEN ALCOHOL
}

\section{SCALES THAT EVALUATE BARRIERS AND ENVIRONMENTAL RESOURCES TO SEEK HELP IN UNIVERSITY STUDENTS WHO DRINK ALCOHOL}

\author{
Martha L. Salazar Garza1', Jessica A. Pantoja Valenciano', Mª de los Ángeles Vacio Muro', Jose A. Ramírez García y Samuel Jurado \\ Cárdenas $^{2}$
}

Departamento de Pscología, Universidad Autónoma de Aguastacalientes. México

²Departamento de Psicología, Universidad Nacional Autónoma de México. México

\section{Abstract}

Studies conducted with university students who drink alcohol show the need to identify the variables that hinder or promote the search for help. The objective of this work was to develop two scales that assess the barriers and environmental resources for seeking help, in Mexican university students who consume alcohol in excess. We worked with 437 students with an average age of 19.85 years, consumers of alcohol in excess, through an instrumental design. Reagents were analyzed by Student's $t$-tests for independent samples, exploratory and confirmatory factor analysis, and the cronbach alpha index. The barrier scale was composed of 9 items in three factors ( $\alpha=.86$ ): standard of morality, absence of social support from parents and social stigma and absence of social support from peers. The resource scale was integrated by 12 items in four factors $(\alpha=.84)$ : health and social support systems, negative consequences, rejection of parents and friends, and rejection of parents and partners. The reliability for the barrier scale was .86 and for the resource scale of .84. In conclusion, two scales were obtained with adequate psychometric characteristics to be used with Mexican university students.

Keywords: Mexican students, excessive alcohol consumption, environmental resources, environmental barriers, helpseeking.

\section{Resumen}

Los estudios realizados con universitarios que beben alcohol muestran la necesidad de identificar las variables que dificultan o promueven la búsqueda de ayuda. El objetivo de este trabajo fue desarrollar dos escalas que evalúan las barreras y los recursos ambientales para la búsqueda de ayuda, en jóvenes universitarios mexicanos que consumen alcohol en exceso. Se trabajó con 437 estudiantes con edad promedio de 19.85 años, consumidores de alcohol en exceso, mediante un diseño instrumental. Los reactivos se analizaron mediante pruebas $t$ de Student para muestras independientes, análisis factorial exploratorio y confirmatorio, y el índice de alfa de cronbach. La escala de barreras quedo compuesta por 9 reactivos en tres factores $(\alpha=.86)$ : estándar de moralidad, ausencia de soporte social por parte de padres y, estigma social y ausencia de soporte social por parte de pares. La escala de recursos se integró por 12 reactivos en cuatro factores $(\alpha=.84)$ : sistemas de salud y soporte social, consecuencias negativas, rechazo de padres y amigos y, rechazo de padres y pareja. La confiabilidad para la escala de barreras fue de .86 y para la de recursos de .84. En conclusión se obtuvieron dos escalas con adecuadas características psicométricas para emplearse con estudiantes universitarios mexicanos.

Palabras clave: Estudiantes mexicanos, consumo excesivo de alcohol, recursos ambientales, barreras ambientales, búsqueda de ayuda. 
El consumo de alcohol entre los jóvenes universitarios es una práctica social común que conlleva efectos positivos como la desinhibición, la convivencia social y las sensaciones placenteras. Estas consecuencias, junto con un consumo moderado no implicarían una preocupación importante; sin embargo, se ha encontrado que los jóvenes subestiman los riesgos asociados al consumo excesivo de alcohol, ya que no lo consideran lo suficientemente riesgoso, minimizando con ello las consecuencias negativas, por lo que suelen pensar que no requieren de ningún tipo de ayuda para cambiar su consumo (Kahi, Abi-Rizk, Hlais \& Adib, 2012; Zuñiga \& Bouzas, 2006).

La Organización Mundial de la Salud (OMS) desarrolló el Cuestionario de Identificación de los Trastornos debidos al Consumo de Alcohol (AUDIT), con la finalidad de que el personal sanitario y profesionales de la salud pudieran identificar, de forma simple, a las personas con un patrón de consumo de alcohol excesivo, es decir un consumo que supone un riesgo o un daño importante para la persona. Una de las razones principales de haber desarrollado el AUDIT fue que la mayoría de bebedores excesivos no estaban diagnosticados, pero presentaban síntomas o problemas que normalmente no se relacionan con la manera en que consumen alcohol. El consumo de alcohol riesgoso supone consecuencias adversas para el propio bebedor o para los demás, pero el bebedor aún no experimenta ningún trastorno. En cambio, el consumo de alcohol perjudicial conlleva consecuencias en la salud física y mental; incluyendo en algunos, consecuencias de tipo social (Babor, Higgins-Biddle, Saunders \& Monteiro, 2001).

Los últimos resultados publicados por la Encuesta Nacional de Consumo de Drogas, Alcohol y Tabaco (ENCODAT) 2016-2017 en México (Instituto Nacional de Psiquiatría Ramón de la Fuente Muñiz, Instituto Nacional de Salud Pública, Comisión Nacional Contra las Adicciones \& Secretaría de Salud, 2017) reportaron que en el último mes, el consumo 2016-2017 también arrojó un incremento en el consumo excesivo de alcohol (beber cinco o más cervezas o cubas de cualquier bebida alcohólica en una o más ocasiones) durante el último mes en adolescentes de 12 a 19 años se duplicó, pasando de $4.3 \%$ a $8.3 \%$. En el caso particular de las mujeres, el incremento fue de más de tres veces (de $2.2 \%$ a $7.7 \%$ ). Sin embargo, el consumo diario y excesivo de alcohol en los hombres aumentó más de 6 veces (de $0.4 \%$ a 2.4\%).

Por otro lado, la ENCODAT reportó que el grupo con mayor consumo per cápita fue el de los 18 a 29 años (7.6 litros), seguido por el grupo de 15 a 17 años (5.9 litros). Además, se encontró que en 2016, los servicios de urgencias hospitalarias fueron solicitados por un $13.5 \%$ de estudiantes. De éstos, las mujeres de 15 a 29 años tuvieron una mayor prevalencia de ingreso, en comparación con los hombres. También se reportó que el grupo de adolescentes 12 a 19 años (48.2\%) y los adultos jóvenes de 18 a 29 años (31.3\%), solicitaron en mayor medida los servicios de las Unidades de Especialidades Médicas - Centro de Atención Primaria en Adicciones (UNEME-CAPAS).

Dado el consumo de alcohol identificado en jóvenes en edad de cursar la universidad y sus consecuencias asociadas, como las descritas previamente, existe interés por parte de los investigadores en identificar las variables asociadas a la búsqueda de ayuda o de tratamiento por parte de los jóvenes, para modificar la manera en que consumen alcohol. En jóvenes mexicanos, se ha identificado una baja aceptación para asistir a tratamiento encaminado a disminuir el consumo de alcohol (Salazar, Avila, Pérez \& Martínez, 2010). En otros países, se ha evidenciado que la búsqueda de ayuda para tratar el consumo de sustancias es menor, en comparación con la búsqueda de ayuda para tratar otros problemas de salud (Blanco, Okuda, Wright, et al., 2008).

Se ha reportado que algunas variables individuales pueden obstaculizar la búsqueda de ayuda, como el hecho de que las personas consideren que por sí mismos, pueden cambiar su consumo excesivo de alcohol (Saunders, Zygowicz \& D'Angelo, 2006), así como considerar que su manera de beber alcohol no es un problema serio (Tucker, Vuchinich \& Rippens, 2004; Tomás-Dols, Valderrama-Zurían, Vidal-Infer, SamperGras, Hernández-Martínez \& Torrijo-Rodrigo, 2007; Wu, Pilowsky, Schlenger \& Hasin, 2007).

Los estudios también han mostrado que algunos factores sociales pueden dificultar la búsqueda de ayuda (George \& Tucker, 1996; Jordan \& Oei, 1989; Weisner \& Matzger, 2002; Jacobson, 2007). Uno de los factores es el temor al estigma y la falta de reconocimiento por parte de personas cercanas de que su consumo de alcohol requiere atención (Blanco, Okuda, Wright, et. al., 2008); 
otros factores son las características de las redes sociales de apoyo, el estigma respecto al consumo excesivo de alcohol en las mujeres, la creencia entre los hombres de que ingresar a un tratamiento atenta contra su masculinidad y las dificultades para acceder a los servicios de salud (Tomás-Dols et al., 2007).

Ante la dificultad de que los jóvenes busquen ayuda para realizar cambios a favor de su salud, se han desarrollado distintos modelos que buscan dar cuenta del proceso que conlleva el establecimiento de conductas saludables. En particular, el modelo propuesto por Schwarzer \& Fuchs (1995) integra variables psicológicas que en la literatura se han visto asociadas con la búsqueda de tratamiento, tales como la autoeficacia percibida, la percepción de riesgo y las expectativas de los individuos, así como por las barreras y los recursos ambientales. Según Bandura (1997; 1999, 2001), el ambiente es todo lo físico y convencionalmente establecido que tiene influencia sobre el individuo. Una barrera puede ser todo aquello que impide al individuo llegar a una meta, pueden ser materiales y sociales. Las barreras son situaciones, condiciones o eventos que se oponen a la agencia personal y que obstaculizan su movilidad estructural en el ambiente. Mientras que un recurso puede ser todo aquello que favorece que el sujeto alcance una meta y también pueden ser materiales y sociales. Los recursos son situaciones, condiciones o eventos que promueven la agencia personal y favorecen su movilidad estructural en el ambiente. La evaluación de barreras o recursos para la búsqueda de ayuda puede realizarse a través de la observación directa, mediante el reporte de los individuos o mediante la construcción de instrumentos de medición; ya sea para problemas de salud en general, salud mental o para el consumo de sustancias.

En el caso particular del campo de las adicciones, George \& Tucker (1996), evaluaron las barreras e incentivos para la búsqueda de ayuda en bebedores problema, a través de una Escala tipo Likert (con valores de 0 al 10), en las que los individuos determinan la influencia de algunos factores sobre la decisión de iniciar un tratamiento o de ingresar a un grupo de AA. La Escala fue desarrollada considerando estudios previos en los que se retomaban modelos médicos para la búsqueda de ayuda por problemas relacionados con el consumo de alcohol. No obstante, los autores no reportan datos sobre la validez o confiabilidad del instrumento. El empleo de la escala con una muestra de 45 adultos bebedores que reportaron problemas con su consumo de alcohol y que habían o no asistido a tratamiento previamente arrojó que los factores considerados como barreras para asistir a tratamiento, entre quienes no lo habían hecho en el pasado, incluían: considerar que su consumo era un problema serio o que lo podían resolver por ellos mismos, la preocupación por ser etiquetados o por la confidencialidad de la información, el costo y la distancia de los tratamientos, la interferencia con las actividades cotidianas y las experiencias negativas anteriores. Mientras que las barreras para asistir a un grupo de AA entre quienes habían asistido a otro tipo de tratamiento en el pasado fueron: las preocupaciones acerca de ser etiquetados, de compartir información en un formato grupal, la creencia de que se tenía un problema que no era tan serio como para asistir a $\mathrm{AA}$, al igual que la distancia, la duración de las sesiones, la interferencia con las responsabilidades, la falta de privacidad, el conocer a otros con problemas por su consumo de alcohol y que no fueron a AA, la orientación espiritual de AA.

Por otra parte, los incentivos para asistir a tratamiento entre quienes no lo habían hecho fueron: los problemas familiares, el aliento de la familia para ingresar a tratamiento, el creer que no podían resolver los problemas por sí mismos, el que hubieran asistido a tratamiento por una orden judicial, problemas en el trabajo o que los colegas hubieran motivado para que se entrara a tratamiento, problemas de salud y conocer a otros que ya habían buscado ayuda o tratamiento. Los incentivos para asistir a AA entre quienes habían asistido a otro tratamiento fueron; las características particulares de los grupos de AA (formato grupal, orientación espiritual, la posibilidad de ayudar a otros, el acceso gratuito, entre otras), los problemas familiares y que la familia alentara el ingreso a AA, considerar que no podrían resolver los problemas por ellos mismos, problemas de salud, conocer a otros que hubieran asistido a AA, problemas en el trabajo o el apoyo de los compañeros para ir a AA, el asistir a las reuniones en lugar de ir a beber.

Cellucci, Krogh \& Vik (2006) desarrollaron una Escala para medir el estigma social relacionado con la búsqueda de ayuda por parte de estudiantes universitarios con una edad promedio de 22 años. La validación de la Escala se realizó mediante análisis factorial exploratorio, obteniendo dos dimensiones que dieron cuenta del 44\% 
de la varianza. La primera dimensión, claramente estaba relacionada con el estigma social, ya que incluía preguntas que reflejaban el desconcierto o la confusión de los individuos por tener que hablar con algún consejero sobre los problemas asociados con su consumo de alcohol, el considerarse débil por buscar ayuda, pensar que los individuos que buscan tratamiento deben ser quienes tienen mayores pérdidas, sentirse como alcohólicos por el hecho de buscar ayuda, sentir vergüenza si otros se enteran de que han hablado con algún profesional acerca del consumo, ver como posibilidad la pérdida del trabajo si otros se enteran de que se tiene problemas relacionados con la manera de beber. Mientras que la segunda dimensión medía aspectos relacionados con la falta de percepción sobre la necesidad de buscar tratamiento. Sin embargo, su uso con una muestra de 133 estudiantes universitarios mostró que el estigma no fue una variable que estuviera correlacionada con la búsqueda de ayuda, en comparación de otras variables evaluadas como la disposición al cambio, el puntaje alcanzado en el AUDIT y con los síntomas de ansiedad y depresión. Si bien esta Escala no mide de manera particular la variable de barreras o recursos, evalúa una de las variables que en la literatura se han mencionado como posible barrera para la búsqueda de ayuda.

No obstante, debido a que los jóvenes mexicanos en edad de cursar la Universidad presentan un patrón de consumo de alcohol riesgoso, es importante desarrollar un instrumento que permita evaluar las barreras tanto personales como sociales que impiden que los jóvenes universitarios busquen ayuda para disminuir su consumo de alcohol y a la vez, permita identificar los recursos o incentivos que los jóvenes identifican. En la literatura no se encontró una Escala específica, sino que más bien, se trata de instrumentos que valoraban diferentes variables que después se correlacionan con la búsqueda de ayuda (Buscemi, Murphy, Martens, McDevitt-Murphy, Pederson \& Skidmore, 2010; Caldeira, Kasperski, Pharm, Vincent, Grady, Wish \& Arria, 2009; Dawson, Goldstein \& Grant, 2012) o bien, instrumentos para evaluar población adulta que presenta consecuencias sociales más problemáticas (George \& Tucker, 1996). Por lo tanto, el objetivo de este trabajo fue construir una escala psicométrica que facilite la evaluación de las barreras y los recursos ambientales, relacionados con la búsqueda de ayuda en estudiantes universitarios que consumen alcohol en exceso.

\section{MÉTODO}

\section{Diseño y participantes}

Estudio instrumental que siguió las normas para el desarrollo y revisión de estudios instrumentales (Carretero-Dios \& Pérez, 2007).

Participaron 437 universitarios (267 hombres y 170 mujeres), con una edad promedio de 19.85 años ( $D T=2.06)$. El 98.9\% reportó estar soltero al momento del estudio, el resto dijo estar casado u omitió el dato.

Los estudiantes estaban inscritos en cuatro universidades públicas (84\%) y una privada (16\%) del Estado de Aguascalientes en México, asociadas al Consejo Interuniversitario Contra las Adicciones (CICA), quienes cursaban del primero al octavo semestre $(M=$ 2.59, $D E=1.83$ ) y fueron seleccionados por conveniencia de un total de 4787 estudiantes, por reportar un consumo riesgoso y dañino de alcohol de 5 o más puntos en el Cuestionario de Identificación de los Trastornos debidos al Consumo de Alcohol [AUDIT] (Medina-Mora \& Carreño, 1998).

\section{Instrumentos}

Cuestionario de Identificación de los Trastornos debidos al Consumo de Alcohol (AUDIT) (Medina-Mora \& Carreño, 1998). Su objetivo es evaluar el consumo riesgoso y dañino de alcohol [CRDA]. Consta de 10 reactivos, cada uno con diferentes opciones de respuesta. Su validez ha sido calculada frente al diagnóstico de consumo perjudicial y de dependencia. En su aplicación con población mexicana se obtuvo un alpha de cronbach de.77. Este cuestionario fue empleado para seleccionar a los estudiantes, considerando un puntaje de 5 o más puntos. Un puntaje de 5 a 7 puntos indica que la persona ha tenido consumos excesivos y algunas consecuencias negativas, por lo que requiere evaluar su consumo y considerar las ventajas de cambiarlo. Los puntajes por arriba de 8 puntos muestran claramente que la persona bebe en exceso y que ya ha tenido varias consecuencias, por lo que requiere hacer un cambio pronto en su manera de tomar alcohol.

Cuestionario de datos sociodemográficos. Recaba información sobre sexo, edad, estado civil, universidad, carrera y semestre/cuatrimestre que cursa el estudiante. 


\section{Procedimiento}

En un primer momento, se realizó una revisión teórica-conceptual de los postulados de la teoría de Bandura que contribuían en la comprensión de las variables de barreras y recursos ambientales. Se dio lectura a diferentes recursos bibliográficos en los que se hacía referencia a las variables que influyen en la adopción de conductas saludables y a la par se celebraron reuniones semanales entre los expertos, a fin de lograr el consenso en las definiciones conceptuales y operacionales.

Se obtuvo un banco de reactivos mediante la técnica de grupos focales (Kitzinger, 1994) con estudiantes universitarios. En total se tuvieron 5 grupos, con la participación total de 48 jóvenes (34 hombres y 14 mujeres), entre los 18 y 25 años de edad, consumidores de alcohol e inscritos en universidades públicas y privadas, de zonas rurales y urbanas del Estado de Aguascalientes, asociadas al CICA. Se determinó un número impar de grupos focales considerando el criterio de desempate o tercero en discordia, al haber la posibilidad de opiniones opuestas entre ellos (Vaugh, Shay \& Sinagub, 1996). Los estudiantes fueron invitados de manera voluntaria, bajo el criterio de que fueran consumidores de alcohol, a quienes se les proporcionó un pase para el cine por su colaboración.

Con la información recabada en los grupos focales se diseñó una propuesta de las escalas. Esta versión se envió a tres jueces expertos en el tema, quiénes contaban con maestría y habían realizado trabajo profesional o de investigación en el área de adicciones. Los jueces consideraron que el formato, el nombre de la escala, las instrucciones y opciones de respuesta eran pertinentes, realizando sugerencias para el cambio en la redacción de algunos reactivos.

Posteriormente, se llevó a cabo un ensayo de evaluación con 45 estudiantes, a partir del cual se identificó que era necesario modificar las opciones de respuesta. Inicialmente las opciones de respuesta eran: Totalmente en desacuerdo, En desacuerdo, De acuerdo y Totalmente de acuerdo. Las cuales se cambiaron por Totalmente en desacuerdo, Poco de acuerdo, Muy de acuerdo y Totalmente de acuerdo, con valores que van desde el 1 al 4 para su calificación.
Con base en la información proporcionada por los grupos focales, la valoración de los jueces y el ensayo de evaluación, la escala de Barreras ambientales quedó representada en 17 reactivos, en tres categorías: estigma social (6 reactivos), estándar de moralidad respecto al consumo de alcohol (7 reactivos) y ausencia de soporte social por parte de personas significativas (4 reactivos). Mientras que, la escala de Recursos ambientales se integró por 30 reactivos, agrupados en cuatro categorías: sistema de salud (seis reactivos), rechazo de significativos (siete reactivos), soporte social por parte de personas significativas ( 12 reactivos) y consecuencias negativas ( 5 reactivos).

Es importante aclarar que los reactivos seleccionados fueron aquellos que alcanzaron el acuerdo del $80 \%$ entre los jueces que evaluaron cada reactivo, respecto con su pertenencia a las variables medidas. También es necesario aclarar que se desarrollaron dos escalas, debido a que en la escala de recursos ambientales se solicita a los individuos que especifiquen las situaciones que favorecen la búsqueda de ayuda. En cambio en la escala de barreras ambientales, se pide a los evaluados que especifiquen las situaciones que obstaculizan la búsqueda de ayuda. En Anexo 1 se muestran las categorías obtenidas, su definición y los reactivos que fueron incluidos en cada categoría; sometidos posteriormente a los análisis estadísticos.

Finalmente, se realizó la aplicación final de manera grupal en formato electrónico, durante los horarios de clase acordados con las universidades. Cinco colaboradores del proyecto asistieron a las instituciones para la evaluación final. Los estudiantes fueron informados al inicio de la evaluación sobre el objetivo y condiciones de la misma, firmando su consentimiento de manera electrónica. La aplicación final se realizó con 4787 estudiantes, sin embargo para los análisis solo se consideraron 437, quienes tuvieron 5 o más puntos en el AUDIT, debido a que este puntaje los ubicaba en la luz amarilla de un semáforo, de acuerdo a una de las maneras en cómo se pueden interpretar los resultados del instrumento, pues seguramente estos jóvenes habían presentado ya alguna consecuencia negativa por su consumo de alcohol.

\section{Análisis de datos}

Los datos obtenidos se analizaron con el paquete estadístico SPSS 20. Los análisis estadísticos de los 
reactivos en ambas escalas consistieron en realizar pruebas $t$ de Student para muestras independientes, considerando que cada reactivo debía alcanzar una significancia igual o menor al .05.

Los reactivos que discriminaron fueron sometidos a las pruebas de esfericidad de Barlett. Se realizó un análisis factorial exploratorio de componentes principales, mediante rotación ortogonal a través del método varimax. Los reactivos seleccionados fueron sometidos al análisis factorial confirmatorio de máxima verosimilitud, a través del módulo AMOS del SPSS versión 20. Finalmente, se obtuvo el índice de confiabilidad mediante al alfa de Cronbach, para cada escala y para sus diferentes factores.

Además, se empleó la prueba $t$ de Student para muestras independientes, con el propósito de analizar las diferencias por sexo (masculino o femenino), tipo de escuela (privada o pública) y puntaje en el AUDIT (Grupo 1: de 5 a 7 puntos, Grupo 2: de 8 o más puntos), para los factores de ambas escalas. Estos análisis fueron considerados para evaluar si existía algún tipo de barrera o recurso ambiental que diferenciara a los hombres y mujeres, a los estudiantes de escuelas públicas y privadas, o por su nivel de consumo de riesgo y dañino de alcohol.

\section{Consideraciones éticas}

De acuerdo con el APA (2017) y el Código Ético del Psicólogo (Sociedad Mexicana de Psicología, 2009) se realizó un balance en cuanto al riesgo/beneficio de los participantes y se identificó que el responder la Escala en desarrollo no genera ningún riesgo para los participantes y el valor potencial científico y social de la generación de dicha herramienta es considerado como trascendente. Si bien, la selección de los participantes se realizó mediante un cuestionario que evalúa si la persona presenta un patrón de consumo de alcohol de riesgo, cuyos resultados pueden poner en riesgo social a los participantes, los investigadores aseguraron el resguardo y confidencialidad de los datos.

Además, se ofreció a los estudiantes participar en una evaluación y retroalimentación más amplia de su consumo de alcohol mediante una página web diseñada para este propósito (Pérez, Ortiz, Vacio \& Salazar, 2016). A su vez, dicha retroalimentación disminuye cualquier riesgo psicológico que pudieran correr los participantes una vez de que son conscientes de que la forma de beber los pone en riesgo. Se descartaron posibles daños físicos.

Adicionalmente, se diseñó un consentimiento informado en el cual se explicaba la finalidad del estudio, el resguardo y protección de sus datos, así como la posibilidad de realizar preguntas durante el estudio, resaltándose los beneficios que podrían recibir al ser evaluados y al participar en el desarrollo de la Escala. Es importante señalar que el estudio fue aprobado por un comité de evaluadores seleccionado por la Dirección de Investigación y Posgrado de la Universidad Autónoma de Aguascalientes, quien otorgó el financiamiento para el proyecto.

\section{RESULTADOS}

Características de Consumo de los Estudiantes que Participaron en la Aplicación Final

De los 437 estudiantes, el $32.3 \%$ tuvo de 5 a 7 puntos en el AUDIT y el $67.7 \%$ tuvo 8 puntos o más. El 66.4\% reportó tomar bebidas alcohólicas de dos a cuatro veces al mes, el 19.9\% dijo hacerlo dos o tres veces por semana, $13.3 \%$ una vez al mes, y el 5\% reportó tomar ocasionalmente. El $52.6 \%$ dijo beber 5 o 6 copas en un día típico o común de los que beben, el $40.3 \%$ dijo beber de 3 a 4 copas y el 7.1\% 1 o 2 copas. Además, el 47.6\% expresó beber 6 o más copas en la misma ocasión de dos a cuatro veces al mes, 32.5\% dijo hacerlo de dos una vez al mes o menos, $12.1 \%$ dos tres veces por semana, $4.1 \%$ diario o casi diario y $3.7 \%$ dijo que nunca bebía en estas cantidades.

\section{Características de Validez y Confiabilidad de la Escala de Barreras Ambientales}

Para la escala de barreras ambientales, la prueba $t$ de Student para muestras independientes mostró que los 17 reactivos discriminaron entre el grupo con las calificaciones más altas y el grupo con las calificaciones más bajas, con una significancia estadística menor a 001 . El alfa de Cronbach arrojó que todos los reactivos tuvieron una correlación reactivo-calificación total mayor a .20. La Tabla 1 muestra los valores $t$ y los índices de correlación para cada reactivo. 
Tabla 1. Análisis de reactivos de la escala de barreras para la búsqueda de ayuda

\begin{tabular}{|c|c|c|}
\hline & $t$ & CR-T \\
\hline $\begin{array}{l}\text { BARR 1.Que me llamen borracho(a) por buscar } \\
\text { ayuda para cambiar mi forma de beber }\end{array}$ & -11.906 & .441 \\
\hline $\begin{array}{l}\text { BARR 2.Que en mi familia se acostumbre a } \\
\text { beber alcohol en exceso frecuentemente }\end{array}$ & -13.919 & .534 \\
\hline $\begin{array}{l}\text { BARR 3. Que mis padres no me apoyen en mi } \\
\text { decisión de buscar ayuda para cambiar mi } \\
\text { forma de beber }\end{array}$ & -13.263 & .502 \\
\hline $\begin{array}{l}\text { BARR 4. Que los jóvenes con los que me junto } \\
\text { consideren normal el beber alcohol en exceso }\end{array}$ & -20.990 & .674 \\
\hline $\begin{array}{l}\text { BARR 5. Que alguno de mis seres queridos beba } \\
\text { alcohol muy a menudo }\end{array}$ & -17.354 & .650 \\
\hline $\begin{array}{l}\text { BARR 6. Que mis amigos(as) me consideren } \\
\text { aburrido(a) por dejar de tomar alcohol }\end{array}$ & -14.702 & .536 \\
\hline $\begin{array}{l}\text { BARR 7. Que los jóvenes con los que me junto } \\
\text { acostumbren beber alcohol en exceso casi } \\
\text { todos los fines de semana }\end{array}$ & -20.797 & .633 \\
\hline $\begin{array}{l}\text { BARR 8. Que en mi familia se permita beber } \\
\text { alcohol en exceso }\end{array}$ & -15.786 & .659 \\
\hline $\begin{array}{l}\text { BARR 9.Que mis padres no me motiven a buscar } \\
\text { ayuda para cambiar mi forma de beber alcohol }\end{array}$ & -14.044 & .565 \\
\hline $\begin{array}{l}\text { BARRR 10. Que a las fiestas a las que acudo se } \\
\text { acostumbre beber alcohol en exceso }\end{array}$ & -20.512 & .656 \\
\hline $\begin{array}{l}\text { BARR 11. Que me critiquen por dejar de tomar } \\
\text { alcohol }\end{array}$ & -15.285 & .640 \\
\hline $\begin{array}{l}\text { BARR 12. Que mis familiares me inviten a beber } \\
\text { alcohol con ellos }\end{array}$ & -15.388 & .516 \\
\hline $\begin{array}{l}\text { BARRR 13. Que mis amigos(as) no apoyen mi } \\
\text { decisión de buscar ayuda para cambiar mi } \\
\text { forma de beber alcohol }\end{array}$ & -16.157 & .630 \\
\hline $\begin{array}{l}\text { BARR 14. Que la gente crea que no puedo } \\
\text { disminuir mi consumo de alcohol por mí } \\
\text { mismo(a) }\end{array}$ & -13.334 & .567 \\
\hline $\begin{array}{l}\text { BARR 15. Que me llamen alcohólico(a) por } \\
\text { buscar ayuda para cambiar mi forma de beber } \\
\text { alcohol }\end{array}$ & -15.574 & .641 \\
\hline $\begin{array}{l}\text { BARR 16. Que mis amigos(as) no me motiven a } \\
\text { buscar ayuda para cambiar mi forma de beber } \\
\text { alcohol }\end{array}$ & -18.644 & .698 \\
\hline $\begin{array}{l}\text { BARR 17. Que mis amigos(as) ya no me } \\
\text { consideren divertido(a) por querer reducir mi } \\
\text { consumo de alcohol }\end{array}$ & -16.325 & .667 \\
\hline
\end{tabular}

Nota: CR-T=Correlación reactivo-calificación total

La prueba de esfericidad de Barlett para esta escala mostró que la matriz de correlación de los reactivos no era idéntica (4.185 $p=\leq .000$ ). Mientras que el puntaje Kaiser-Meyer-Olkin alcanzó un valor significativo meritorio (.93). El análisis factorial exploratorio de componentes principales, mediante el método Varimax con rotación ortogonal mostró la presencia de tres factores, conservándose 13 reactivos con cargas factoriales igual o mayor a .40; eliminando 4 reactivos que cargaron en dos factores (BARR 5, BARR 6, BARR 8 y BARR 11). Enseguida, con el fin de corroborar la estructura factorial obtenida, se ejecutó un análisis factorial confirmatorio mediante ecuaciones estructurales, empleando el paquete AMOS, a través del cual se eliminaron cuatro reactivos más (BARR 1, BARR 10, BARR 12 y BARR 15). Los resultados mostraron que el modelo obtuvo un buen ajuste $X^{2}=1.939, \mathrm{gl}=32, p=.001$, RMSEA= $.046, \mathrm{NFI}=.961, \mathrm{RFI}=.946, \mathrm{IFI}=.981, \mathrm{TLI}=.973, \mathrm{CFI}=.981$ (Véase Figura 1).

La escala de barreras ambientales quedó integrada por 9 reactivos agrupados en tres dimensiones: 1) Estándar de moralidad respecto al consumo de alcohol, constituida por tres reactivos (BARR 4, BARR 5 y BARR 7), con un índice de confiabilidad de .80; sus reactivos muestran que el individuo se rodea de amigos y familiares que beben alcohol en exceso y de manera frecuente. 2) Ausencia de soporte social por parte de pares y estigma social: conformada por cuatro reactivos (BARR 13, BARR 14, BARR 16 y BARR 17), con un índice de confiablidad de .82; los cuales sugieren que los jóvenes consideran que sus amigos no los motivan ni están de acuerdo en que busquen ayuda y consideran que pueden ser vistos por éstos como personas que no saben divertirse si lo hacen y 3) Ausencia de soporte familiar, integrada por dos reactivos (BARR 3 y BARR 9), alcanzando un índice de confiabilidad de .66, sugiriendo que los padres de los individuos no motivan ni apoyan la idea de buscar ayuda. Los tres factores tuvieron una confiabilidad adecuada pues se encontraron alrededor o más arriba del .70. El índice confiabilidad para la escala total fue de .86 .

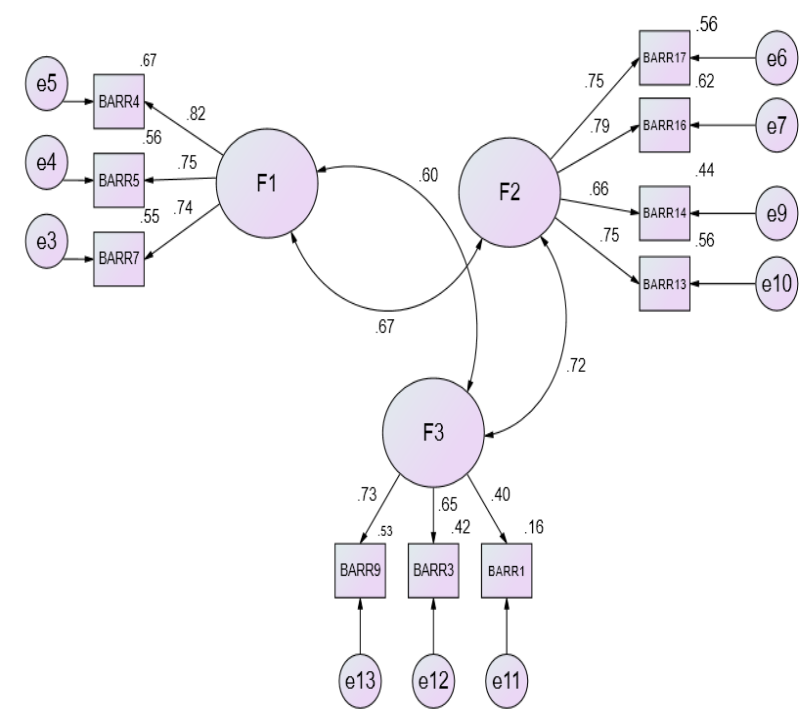

Figura 1. Cargas factoriales para cada reactivo y correlación con el resto de la escala. F1= Estándar de moralidad respecto al consumo de alcohol, F2=Ausencia de soporte social por parte de pares y estigma social, F3=Ausencia de soporte familiar. 


\section{Características de Validez y Confiabilidad de la Escala de Recursos Ambientales}

Los análisis de la escala de recursos ambientales mediante la prueba $t$ de Student para muestras independientes, mostró que los 30 reactivos discriminaron entre el grupo con las calificaciones más altas y el grupo con las calificaciones más bajas, con una significancia estadística menor a .001. El alfa de cronbach arrojó que todos los reactivos tuvieron un índice de la correlación reactivo-calificación total mayor a .20. La Tabla 2 muestra los valores $t$ y los índices de correlación para cada reactivo.

Tabla 2. Análisis de reactivos de la escala de recursos para la búsqueda de ayuda

\begin{tabular}{|c|c|c|}
\hline & $t$ & CR-T \\
\hline $\begin{array}{l}\text { REC 1. Que mis profesores me animen a } \\
\text { reducir mi consumo de alcohol para } \\
\text { mejorar mi aprovechamiento en clase. }\end{array}$ & -14.786 & .578 \\
\hline $\begin{array}{l}\text { REC 2. La difusión de consecuencias de } \\
\text { beber alcohol en exceso, a través de } \\
\text { anuncios publicitarios }\end{array}$ & -14.464 & .578 \\
\hline $\begin{array}{l}\text { REC 3. Que mis papás no estén de } \\
\text { acuerdo con mi forma de beber alcohol }\end{array}$ & -11.405 & .496 \\
\hline $\begin{array}{l}\text { REC 4. Que alguien que haya } \\
\text { experimentado consecuencias negativas a } \\
\text { causa de su forma de beber alcohol, me } \\
\text { oriente para buscar ayuda }\end{array}$ & -16.560 & .631 \\
\hline $\begin{array}{l}\text { REC 5. Que mis amigos(as) se quejen de } \\
\text { mi forma de beber cuando salimos }\end{array}$ & -15.854 & .599 \\
\hline $\begin{array}{l}\text { REC 6. Que uno de mis seres queridos se } \\
\text { enferme o tenga un accidente a causa de } \\
\text { su manera de beber alcohol }\end{array}$ & -15.210 & .478 \\
\hline $\begin{array}{l}\text { REC 7. La cercanía de un servicio de salud } \\
\text { al lugar donde vivo }\end{array}$ & -16.648 & .606 \\
\hline $\begin{array}{l}\text { REC 8. Que mis papás me animen a } \\
\text { cambiar mi forma de beber alcohol }\end{array}$ & -17.988 & .679 \\
\hline $\begin{array}{l}\text { REC 9. Que mis amigos(as) me digan a } \\
\text { dónde puedo acudir para cambiar mi } \\
\text { forma de beber alcohol }\end{array}$ & -21.801 & .717 \\
\hline $\begin{array}{l}\text { REC 10. Que mis papás me digan a dónde } \\
\text { puedo acudir para cambiar mi forma de } \\
\text { beber alcohol }\end{array}$ & -23.492 & .726 \\
\hline $\begin{array}{l}\text { REC 11. Que mis papás me regañen } \\
\text { constantemente por beber alcohol en } \\
\text { exceso }\end{array}$ & -14.500 & .576 \\
\hline $\begin{array}{l}\text { REC 12. Tener un accidente de tránsito a } \\
\text { causa de mi forma de beber alcohol }\end{array}$ & -11.223 & .386 \\
\hline $\begin{array}{l}\text { REC 13. La difusión de información sobre } \\
\text { los servicios de salud a los que puedo } \\
\text { acudir para cambiar mi manera de beber } \\
\text { alcohol }\end{array}$ & -19.848 & .684 \\
\hline $\begin{array}{l}\text { REC 14. Que mi novio(a) me diga dónde } \\
\text { puedo acudir para cambiar mi forma de } \\
\text { beber }\end{array}$ & -23.488 & .724 \\
\hline $\begin{array}{l}\text { REC 15. Que mis amigos(as) ya no quieran } \\
\text { invitarme a fiestas porque bebo } \\
\text { demasiado alcohol }\end{array}$ & -19.113 & .607 \\
\hline
\end{tabular}

Tabla 2. Análisis de reactivos de la escala de recursos para la búsqueda de ayuda (continuación)

\begin{tabular}{|c|c|c|}
\hline & $t$ & CR-T \\
\hline $\begin{array}{l}\text { REC 16. Que mis papás me sugieran que } \\
\text { busque ayuda para cambiar mi forma de } \\
\text { beber alcohol }\end{array}$ & -25.462 & .749 \\
\hline $\begin{array}{l}\text { REC 17. Que alguien importante para mí } \\
\text { muera a causa de su manera de beber } \\
\text { alcohol }\end{array}$ & -13.196 & .428 \\
\hline $\begin{array}{l}\text { REC 18. Que mi novio(a) me sugiera que } \\
\text { busque ayuda para cambiar mi forma de } \\
\text { beber alcohol }\end{array}$ & -21.074 & .717 \\
\hline $\begin{array}{l}\text { REC 19. La cercanía de un servicio de salud } \\
\text { al lugar donde estudio }\end{array}$ & -20.029 & .682 \\
\hline $\begin{array}{l}\text { REC 20. Que mis papás me amenacen con } \\
\text { correrme de la casa por mi forma de } \\
\text { beber }\end{array}$ & -13.024 & .483 \\
\hline $\begin{array}{l}\text { REC 21. Que mi novio(a)se queje } \\
\text { constantemente de que bebo alcohol en } \\
\text { exceso }\end{array}$ & -23.698 & .667 \\
\hline $\begin{array}{l}\text { REC 22. Que mis amigos(as) me animen a } \\
\text { cambiar mi forma de beber alcohol }\end{array}$ & -25.347 & .767 \\
\hline $\begin{array}{l}\text { REC 23. Tener dinero para acceder a un } \\
\text { servicio de salud }\end{array}$ & -15.277 & .592 \\
\hline $\begin{array}{l}\text { REC 24. Que mi novio(a) me diga que } \\
\text { podemos terminar debido a mi forma de } \\
\text { beber alcohol }\end{array}$ & -19.453 & .597 \\
\hline $\begin{array}{l}\text { REC } 25 \text {. Ser despedido del trabajo a causa } \\
\text { de mi forma de beber alcohol }\end{array}$ & -16.513 & .560 \\
\hline $\begin{array}{l}\text { REC 26. Que mis profesores me animen a } \\
\text { reducir mi consumo de alcohol para evitar } \\
\text { reprobar una materia }\end{array}$ & -22.876 & .715 \\
\hline $\begin{array}{l}\text { REC } 27 . \text { Que mis amigos(as) me sugieran } \\
\text { que busque ayuda para cambiar mi forma } \\
\text { de beber alcohol }\end{array}$ & -28.061 & .802 \\
\hline $\begin{array}{l}\text { REC 28. Reprobar materias a causa de mi } \\
\text { forma de beber }\end{array}$ & -15.167 & .510 \\
\hline $\begin{array}{l}\text { REC 29. El bajo costo de un servicio de } \\
\text { salud }\end{array}$ & -19.957 & .669 \\
\hline $\begin{array}{l}\text { REC 30. Que mi novio(a) me anime a } \\
\text { cambiar mi forma de beber alcohol }\end{array}$ & -21.106 & .705 \\
\hline
\end{tabular}

La prueba de esfericidad de Barlett para esta escala arrojó que la matriz de la correlación de los reactivos no era idéntica (8579.252 $p=\leq .000$ ) y el puntaje KaiserMeyer-Olkin alcanzó un valor significativo meritorio (.94). El análisis factorial exploratorio mostró la presencia de cuatro factores, conservándose 25 reactivos con cargas factoriales igual o mayor a .40; encontrándose que 5 reactivos cargaron en dos factores (REC 1, REC 2, REC 4, REC 8 y REC 25), los cuales fueron eliminados. El análisis factorial confirmatorio mediante ecuaciones estructurales, empleando el paquete AMOS, descartó trece reactivos más (REC 7, REC 9, REC 10, REC 13, REC 14, REC 15, REC 16, REC 18, REC 19, REC 22, REC 26, REC 28 y REC 30). Los resultados mostraron que el modelo obtuvo un buen ajuste $X^{2}=1.855, \mathrm{gl}=48, p=.000$, RMSEA $=.044$, 
$\mathrm{NFI}=.959, \mathrm{RFI}=.943, \mathrm{IFI}=.981, \mathrm{TLI}=.973, \mathrm{CFI}=.980$. (Véase Figura 2).

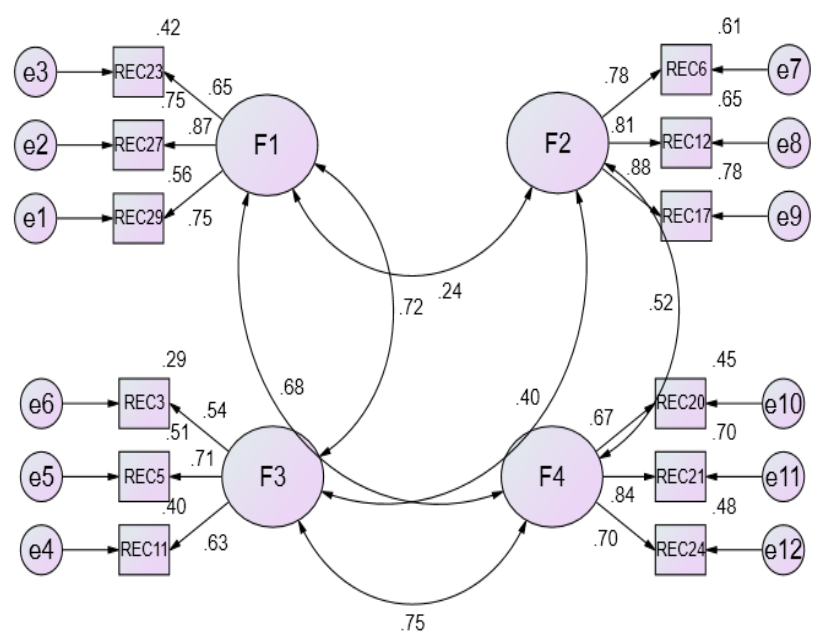

Figura 2. Cargas factoriales para cada reactivo y correlación con el resto de la escala. F1 = Acceso a sevicios de salud y soporte social informacional, F2=Consecuencias negativas graves, F3=Rechazo de padres y amigos; F4=Rechazo de padres y pareja.

De esta forma, la escala de recursos ambientales para la búsqueda de ayuda quedó integrada por 12 reactivos divididos en cuatro dimensiones: 1) Acceso a servicios de salud $y$ soporte social informacional, compuesta por tres reactivos (REC 23 REC 27 y REC 29), con un índice confiabilidad de .80 , los cuales muestran que los individuos consideran que contar con dinero y un bajo costo de los servicios, en conjunto con la sugerencia de buscar ayuda por parte de amistades, facilitan la búsqueda de ayuda; 2) Consecuencias negativas graves, conformada por tres reactivos (REC 6, REC 12 y REC 17), con un índice de confiabilidad de .86, los cuales sugieren que tener de manera directa un accidente de tránsito o que algún familiar o persona cercana, enferme o se muera debido al consumo de alcohol, facilita la búsqueda de ayuda; 3) Rechazo de padres y amigos, integrada por tres reactivos (REC 3, REC 5 y REC 11), con índice de confiabilidad de .66, evidenciando que el desacuerdo de los padres sobre cómo se bebe, el recibir regaños constantes por parte de los mismos y quejas por parte de los amigos en momentos de convivencia, promueve la búsqueda de ayuda y 4) Rechazo de padres y pareja, integrada por tres reactivos (REC 20, REC 21 y REC 24), con un índice de confiabilidad de.77, lo que indica que recibir quejas sobre la manera de beber o advertencias de concluir la relación por parte de la pareja, así como la advertencia por parte de los padres de ser expulsados del hogar, también suscita la búsqueda de ayuda. De igual manera, todos los factores tuvieron una confiabilidad aceptable cercana o por arriba del .70. El índice total de confiabilidad para esta escala fue de .84 .

\section{Diferencias por Sexo, Tipo de Escuela y Puntaje en el AUDIT}

La prueba $t$ de Student de muestras independientes mostró que entre los tres factores de la escala de barreras ambientales, no se encontraron diferencias estadísticamente significativas entre hombres y mujeres: 1) Estándar de moralidad respecto al consumo de alcohol $(t=.818, g l=435, p=.414), 2$ ) Ausencia de soporte social de pares y estigma social ( $t=.893, g l=435, p=.373), 3$ ) Ausencia de soporte familiar ( $t=.482, g /=435, p=.630$ ). Tampoco se encontraron diferencias estadísticas significativas por tipo de escuela: 1) Estándar de moralidad respecto al consumo de alcohol $(t=-.906, g l=$ $435, p=.366), 2$ ) Ausencia de soporte social de pares y estigma social $(t=.916, g l=435, p=.360), 3)$ Ausencia de soporte familiar $(t=.073, g /=435, p=.942)$. Aunque si se encontraron diferencias estadísticas significativas en cada dimensión considerando el puntaje en el AUDIT: 1) Estándar de moralidad respecto al consumo de alcohol $(t=-5.910, g /=435, p=.000), 2$ ) Ausencia de soporte social de pares y estigma social $(t=-4.711, g l=435, p=.000), 3$ ) Ausencia de soporte familiar $(t=-3.165, g l=435, p=.002)$ (Véase Figura 3).

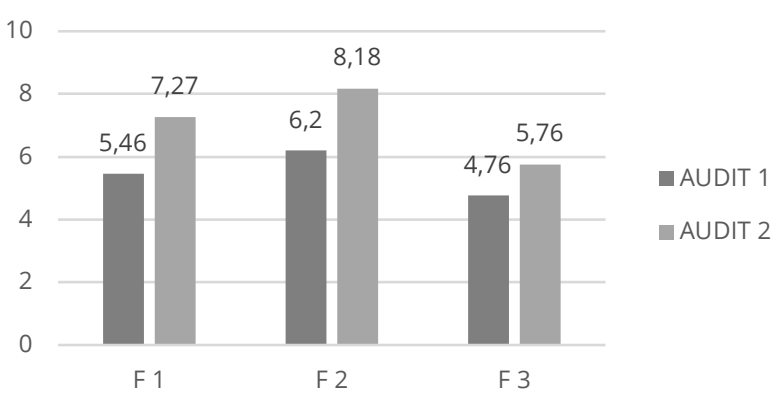

Figura 3. Puntuaciones promedio para los factores de la escala de barreras considerando la puntuación en el AUDIT. F1=Estándar de moralidad respecto al consumo de alcohol, F2= Ausencia de soporte social por parte de pares y estigma social y F3=Ausencia de soporte familiar.

Mientras que para la escala de recursos ambientales, la prueba $t$ de Student de muestras independientes no arrojó diferencias estadísticamente significativas en las cuatro dimensiones por sexo: 1) Acceso a servicios de salud y soporte social informacional $(t=-1.420, g /=435, p=.156), 2$ ) Consecuencias negativas graves $(t=-.752, g l=435, p=.453), 3)$ Rechazo de padres $y$ amigos $(t=-.301, g l=435, p=.764)$ y 4$)$ Rechazo de padres 
y pareja $(t=.315, g l=435, p=.753)$. Tampoco se encontraron diferencias estadísticas significativas por tipo de escuela: 1) Acceso a servicios de salud y soporte social informacional $(t=.135, g /=435, p=.893), 2)$ Consecuencias negativas graves $(t=-.038, g l=435, p=.970), 3)$ Rechazo de padres y amigos $(t=.237, g l=435, p=.813)$ y 4$)$ Rechazo de padres y pareja $(t=.140, g l=435, p=.889)$. Ni por el puntaje en el AUDIT 1) Acceso a servicios de salud y soporte social informacional ( $t=1.205, g l=435, p=.229)$, 2) Consecuencias negativas graves $(t=.666, g /=435, p=$ $.506), 3)$ Rechazo de padres y amigos ( $t=1.411, g /=435$, $p=.159)$ y 4$)$ Rechazo de padres y pareja $(t=.177, g l=435$, $p=.860)$.

\section{DISCUSIÓN}

El trabajo realizado permitió obtener dos escalas con características psicométricas aceptables, una que evalúa las barreras ambientales para la búsqueda de ayuda y otra que evalúa los recursos ambientales para la búsqueda de ayuda, en el cambio del consumo de alcohol, con estudiantes universitarios mexicanos (Véase Anexo 2).

La revisión de los postulados de la Teoría Cognitiva Social, a través del modelo propuesto por Schwarzer y Fuchs (1995), así como la evaluación de jueces expertos, permitió la construcción de reactivos válidos conceptualmente. En tanto que, la implementación de los grupos focales mostró una descripción de las variables ambientales (barreras o recursos), que desde la opinión de los jóvenes universitarios, facilitan e impiden la búsqueda de ayuda para cambiar el consumo de alcohol, teniendo así reactivos con relevancia cultural.

Las dimensiones que integran la escala de barreras ambientales coindicen con los estudios en los que se reporta que la falta de reconocimiento por parte de personas significativas acerca de que se requiere cambiar el consumo de alcohol y el estigma social, son variables sociales que no favorecen la búsqueda de ayuda respecto al consumo de alcohol (Blanco, et al., 2008; Cellucci, et al., 2006), como para los problemas de salud mental (Olivari \& Guzmán-González, 2018). Sin embargo, a diferencia de lo reportado por Cellucci, Krogh \& Vik (2006), el estigma social en este estudio hace referencia al hecho de ser considerado como poco divertido por parte de los amigos y no al temor de ser etiquetado como alcohólico, sentirse débil, sentir vergüenza por buscar ayuda o temor de perder el trabajo. Posiblemente las diferencias puedan deberse a que estos autores hicieron sus estudios con estudiantes universitarios de otro país y con un promedio de mayor edad.

Por su parte, la dimensión de estándar de moralidad de la escala de barreras ambientales, no ha sido reportada previamente en la literatura del tema, pero se puede relacionar con los hallazgos de los estudios que reportan que el aumento en las prevalencias de consumo entre los individuos, posiblemente se relaciona con la alta tolerancia que se tiene al consumo de alcohol entre la población mexicana (Instituto Nacional de Psiquiatría Ramón de la Fuente Muñiz, Instituto Nacional de Salud Pública \& Secretaría de Salud, 2011).

Por otra parte, las dimensiones encontradas para la escala de recursos, son consistentes con las dimensiones que se identificaron en la escala de barreras ambientales, así como con los estudios en los que se reconoce como incentivos para la búsqueda de ayuda, el apoyo social por parte de significativos y la experimentación de consecuencias negativas del propio sujeto o en personas cercanas (Blanco, et al., 2008; George \& Tucker, 1996; Tomás-Dols, et al., 2007). Sin embargo, a diferencia de investigaciones previas (George \& Tucker, 1996), el costo y el tener dinero para acudir a tratamiento, fueron considerados como recursos para buscar ayuda y no como barreras, por parte de los estudiantes universitarios evaluados. Aunque cabe mencionar que los estudios en los que se reportaron estos indicadores como barreras para la búsqueda de ayuda, fueron realizados con adultos. Lo que posiblemente refleje que éstos si consideran el uso del dinero y su costo para acudir a tratamiento, debido a que tienen que cubrir otros gastos como parte de su rol o etapa de desarrollo.

También es importante resaltar que la construcción de la escala permitió encontrar las similitudes y diferencias con los eventos o situaciones que se pueden presentar en adultos y jóvenes. Las situaciones que promovieron la búsqueda de ayuda al igual que en los adultos del estudio realizado por George \& Tucker (1996), fueron tener problemas familiares y el aliento de la familia. Mientras que las diferencias muestran que no se considera como un recurso ambiental para buscar ayuda en la población de universitarios la percepción que se tiene sobre el consumo, haber recibido alguna orden 
judicial, tener problemas en el trabajo o con los colegas, problemas de salud y conocer a otros que ya hubieran recibido algún tipo de ayuda por su consumo de alcohol. En tanto que las barreras ambientales reportadas por los jóvenes del estudio, al igual que los adultos, fueron el temor de ser etiquetados y el costo de los servicios. En tanto que los estudiantes universitarios no consideraron como barreras ambientales la percepción que tienen sobre su consumo, la confidencialidad o la falta de privacidad de la información en los tratamientos, ni la interferencia con otras actividades. Diferencias que posiblemente se asocien con las actividades o el nivel de deterioro de los individuos debido al consumo de alcohol.

Con relación a lo anterior, también es importante resaltar que tanto los jóvenes como los adultos consideran como recursos para buscar ayuda, la ocurrencia de consecuencias negativas, aunque sean de distinta naturaleza. Los reactivos que permanecieron en la dimensión de consecuencias negativas de la escala de recursos ambientales, hacen referencia al hecho de que el propio estudiante tenga un accidente o que alguien cercano enferme o muera debido al consumo de alcohol, mientras que los adultos del estudio de George \& Tucker (1996) hicieron referencia a problemas de salud. Lo que puede deberse al hecho de que es poco probable que los jóvenes experimenten problemas de salud, pero si accidentes. Algunos estudios realizados en México reportan un porcentaje considerable (13.5\%) de jóvenes que llegan los servicios de urgencias, asociados al consumo de alcohol (Instituto Nacional de Psiquiatría Ramón de la Fuente Muñiz, Instituto Nacional de Salud Pública, Comisión Nacional Contra las Adicciones \& Secretaría de Salud, 2017).

Ahora bien, es importante visualizar que los reactivos que al final permanecen en cada una de las escalas corresponden a todas las categorías encontradas con los grupos focales y con los jueces expertos, aún y cuando estos se agruparon de manera distinta, pues podemos encontrar reactivos de más de una categoría en una dimensión. Lo que posiblemente refleje que algunas situaciones se tienen que presentar en conjunto para favorecer u obstaculizar la búsqueda de ayuda. Por ejemplo, el contar con el apoyo de los amigos y tener los recursos económicos para hacerlo, facilita la búsqueda de ayuda. Mientras que la falta de apoyo de los amigos y el ser etiquetados por estos como aburridos, es un obstáculo para la búsqueda de ayuda.
Por otra parte, es importante reconocer que aunque en las categorías identificadas inicialmente se incluyeron diferentes agentes de socialización, solamente los reactivos en los que se hace referencia a los padres, los amigos y pareja son los que permanecen en las escalas finales. Sobresaliendo el papel de los padres, ya que hubo un mayor número de dimensiones que incluía reactivos en los que se describen diferentes respuestas que pueden tener los padres ante el consumo de alcohol de los jóvenes.

Finalmente, los resultados acerca de las diferencias entre los estudiantes considerando su puntaje en el AUDIT, muestran que posiblemente los universitarios que tienen un consumo más riesgoso y perjudicial de alcohol, perciben en mayor medida, contextos sociales en los que se espera que beban en exceso y en los que pueden ser juzgados por buscar ayuda, así como mayor afectación en sus redes de apoyo, lo que se traduce en una falta de apoyo social por parte de sus amigos o padres para buscar ayuda.

Una de las limitaciones del estudio es que no se determinaron otras fuentes de validez, además de la de constructo y factorial. Por lo que, sería importante en estudios futuros buscar otras evidencias para la validez concurrente del instrumento. Además no se consideraron diferencias entre grupos de jóvenes que pudieran en el pasado haber buscado ayuda, como en los estudios realizados con adultos, aún y cuando fuera algún tipo de ayuda informal. Lo que hubiera permitido analizar si existía mayor puntaje por tipo de barrera o recurso, entre los jóvenes que nunca hubieran buscado ayuda y quienes si lo hubieran hecho.

\section{Conclusiones}

En conclusión, la construcción de las escalas permitió obtener dos instrumentos válidos y confiables para la evaluación de las barreras y recursos ambientales relacionados con la búsqueda de ayuda, en jóvenes universitarios mexicanos que presentaron consumo de alcohol de riesgo y perjudicial. Las escalas pueden emplearse con fines de investigación en estudios en los que se busque identificar las variables, ambientales o de otro tipo, que puedan estar asociadas a la búsqueda de ayuda o de tratamiento en jóvenes que consumen alcohol en exceso, para ganar una mejor comprensión del fenómeno, ya que la mayoría de los estudios hacen referencia a variables sociodemográficas, variables de 
tipo individual o evalúan una sola variable que podríamos considerar como ambiental, tal como sería la presión social (Buscemi, Murphy, Martens, McDevitt-Murphy, Pederson \& Skidmore, 2010; Caldeira, Kasperski, Pharm, Vincent, Grady, Wish \& Arria, 2009; Dawson, Goldstein \& Grant, 2012). Por otro lado, el uso de esta escala con fines preventivos podría consistir en emplear sus resultados para el desarrollo de campañas de comunicación o de gestión social, que promuevan la búsqueda de ayuda a fin de disminuir el consumo excesivo de alcohol entre los jóvenes universitarios. Es decir, se podría identificar en los grupos evaluados, cuál es la dimensión de recursos o barreras ambientales que tiene mayor relevancia para la búsqueda de ayuda y con base en ésta, generar la difusión o estrategias que apoyen a los jóvenes.

\section{Agradecimientos}

Se agradece a la Universidad Autónoma de Aguascalientes que otorgó el financiamiento para la realización de este estudio. Así como a todos los estudiantes de pregrado y posgrado que contribuyeron con sus observaciones y trabajo de campo. Finalmente, también se agradece a las escuelas de educación superior asociadas al Consejo Interuniversitario Contra las Adicciones (CICA) del Estado de Aguascalientes, por las facilidades otorgadas para la ejecución del estudio.

\section{Financiación}

Universidad Autónoma de Aguascalientes. Proyecto PIPS14-1, Desarrollo de Estrategias para Promover la Búsqueda de Ayuda en Jóvenes Universitarios que Consumen Alcohol en Exceso.

\section{REFERENCIAS}

American Psychological Association (2017). Ethical Principles of Psychologists and Code of Conduct. Washington, EUA: Recuperado de http://www.apa.org/ethics/code/index.aspx.

Babor, T. F., Higgins-Biddle, J. C., Saunders, J. B., \& Monteiro, M. G. (2001). Cuestionario de Identificación de los Transtornos debidos al Consumo de Alcohol. Ginebra: OMS

Bandura, A. (1997). Self-efficacy: The exercise of control. New York: W. G. Freeman.

Bandura, A. (1999). Social cognitive theory: An agentic perspective. Asian Journal of Social Psychology, 2, 21-41.
Bandura, A. (2001). Social cognitive theory: An agentic perspective. Annual Review Psychology, 52, 1-26.

Blanco, C., Okuda, M., Wrigh, T. C., et al. (2008). Mental Health of College Students and Their Non-College-Attending Peers: Results From the National Epidemiologic Study on Alcohol and Related Conditions. Archives of General Psychiatry, 65(12), 1429-1437. doi:10.1001/archpsyc.65.12.1429

Buscemi, J., Murphy, J. G., Martens, M. P., McDevitt-Murphy, M. E. Pederson, A. A., \& Skidmore, J. R. (2010). Help-Seeking for AlcoholRelated Problems in College Students: Correlates and Preferred Resources. Psychology of Addictive Behaviors, 24(4), 571-580. doi:10.1037/a0021122

Caldeira, K. M., Kasperski, S. J., Sharma, Pharm, B., Vincent, K. B., O'Grady, K. E., Wish, E. D., \& Arria, A. M. (2009). College Students Rarely Seek Help Despite Serious Substance Use Problems. Journal of Substance Abuse Treatment, 37(4), 368-378. doi:10.1016/j.jsat.2009.04.005

Carretero-Dios, H., \& Pérez, C. (2007). Standards for the development and review of instrumental studies: Considerations about test selection in psychological research. International Journal of Clinical and Health Psychology. 7(3), 863-882.

Cellucci, T., Krogh, J., \& Vik, P. (2006). Help Seeking for Alcohol Problems in a College Population. The Journal of General Psychology, I33(A), 421 433.

D. A., Goldstein, R. B., \& Grant, B. F. (2012). Factors Associated With First Utilization of Different Types of Care for Alcohol Problems. Journal of Studies on Alcohol and Drugs, 73, 647-656.

George, A. A., \& Tucker, J. A. (1996). Help-seeking for alcohol-related problems: social contexts surrounding entry into alcoholism treatment or Alcoholics Anonymous. Journal of Studies on Alcohol, 57(4), 449-457.

Instituto Nacional de Psiquiatría Ramón de la Fuente Muñiz, Instituto Nacional de Salud Pública \& Secretaría de Salud (2012). Encuesta Nacional de Adicciones 2011: Reporte de Alcohol. México, D.F.: INPRFM.

Instituto Nacional de Psiquiatría Ramón de la Fuente Muñiz, Instituto Nacional de Salud Pública, Comisión Nacional Contra las Adicciones \& Secretaría de Salud (2017). Encuesta Nacional de Consumo de Drogas, Alcohol y Tabaco 2016-2017: Reporte de Alcohol. Ciudad de México, México: INPRFM.

Jacobson, A. (2007). Characteristics and processes of treatment-seeking for alcohol problems. Sweden: Kompendiet, Göteborg.

Jordan, C. M., \& Oei, T. P. (1989). Help-seeking behavior in problem drinkers: A review. British Journal Addictions, 84, 979-988.

Kahi, E., Abi-Rizk, G., Hlais, S. A. \& Adib, S.M. (2012). Health-care-seeking behavior among university students in Lebanon H.A. Eastern Mediterranean Health Journal. 18(6), 598-606.

Kitzinger, J. (1994). The methodology of Focus Groups: the importance of interaction between research participants. Sociology of Health \& IIIness, 16, 103-121. doi:10.1111/1467-9566.ep11347023 
Medina-Mora, M. E. Carreño, S., \& De la Fuente, R. (1998). Experience with the alcohol use disorders identification test [AUDIT] in Mexico. Recent Developments in Alcoholism, 24, 383-396.

Olivari, C., \& Guzmán-González, M. (2018). Adaptación y validación del cuestionario de barreras para la búsqueda de ayuda para problemas de salud mental en adolescentes chilenos. Universitas Psychologica, 17(1), 1-12. doi:10.11144/Javeriana.upsy17-1.avcb

Pérez, C. J. L., Ortiz, N. S. J., Vacio, M. M. A., \& Salazar, G. M. L. (2016). Percepción de una intervención en modalidad electrónica por universitarios que consumen alcohol. Caleidoscopio. 34, 77-93.

Salazar, G. M., Ávila, H. O., Pérez, J. L., \& Martínez, M. K. (2010). Detección temprana de jóvenes universitarios en riesgo por su consumo de alcohol. Revista de Investigación y Ciencia, 50, 40-46.

Saunders, S. M., Zygowicz, M. S., \& D' Angelo, B. R. (2006). Person-related and treatment-related barriers to alcohol treatment. Journal of Substance Abuse Treatment, 30, 261-270.

Schwarzer, R., \& Fuchs, R. (1995). Modificación de las conductas de riesgo y adopción de conductas saludables: el rol de las creencias de autoeficacia. En: Bandura A, editor. Auto-Eficacia: Cómo afrontamos los cambios de la sociedad actual. España: Desclée de Brouwer; p. 223244.

Sociedad Mexicana de Psicología (2009). Código Ético del Psicólogo. México: Trillas.

Tomás-Dols, S., Valderrama-Zurían, J. C., Vidal-Infer, A., Samper-Gras, T., Hernàndez-Martínez, M. C., \& Torrijo-Rodrigo, M. J. (2007). Género y barreras de accesibilidad al tratamiento en pacientes adictos al alcohol de la Comunidad Valenciana. Adicciones, 19(2), 169-178.

Tucker, J. A., Vuchinich, R. E., \& Rippens, J. A. (2004). A factor analytic study of influences on patterns of help-seeking among treated and untreated alcohol dependent persons. Journal of Substance Abuse Treatment, 26, 237-242.

Vaughn, S., Shay, S. J. \& Sinagub, J. M. (1996). Focus group interviews in education and psychology. United States of America: Sage Publications.

Weisner, C., \& Matzger, H. (2002). A prospective study of the factors influencing entry to alcohol and drug treatment. Journal of Behavioral Health Services \& Research, 29 (2), 126-137.

Wu, L. T., Pilowsky, D. J., Schlenger, W. E. \& Hasin, D. (2007). Alcohol use disorders and the use of treatment services among college-age young adults. Psychiatric Services, 58(2), 192-200.

Zuñiga, A. \& Bouzas, A. (2006). Consumo de alcohol en los adolescentes: percepción de consecuencias positivas y negativas. Revista Mexicana de Psicología, 23(1), 97-110. 
ANEXO 1. Categorías, definiciones y banco de reactivos obtenidos en los grupos focales y mediante jueces expertos para ambas escalas

\section{REACTIVOS SUESCALA BARRERAS}

\begin{tabular}{|c|c|c|}
\hline CATEGORÍA & DEFINICIÓN & REACTIVOS \\
\hline Estigma social & $\begin{array}{l}\text { El sujeto es susceptible de ser "etiquetado" por } \\
\text { buscar ayuda para cambiar su consumo de alcohol. }\end{array}$ & $\begin{array}{l}\text { 1. Que mis amigos(as) me consideren aburrido(a) por dejar } \\
\text { de tomar. } \\
\text { 2. Que mis amigos(as) ya no me consideren divertido(a) por } \\
\text { querer reducir mi consumo de alcohol. } \\
\text { 3. Que me critiquen por dejar de tomar. } \\
\text { 4. Que me llamen borracho(a). } \\
\text { 5. Que me llamen alcohólico(a). } \\
\text { 6. Que la gente crea que no puedo disminuir mi consumo } \\
\text { por mí mismo(a). }\end{array}$ \\
\hline $\begin{array}{l}\text { Estándar de moralidad } \\
\text { respecto al consumo de } \\
\text { alcohol }\end{array}$ & $\begin{array}{l}\text { El sujeto vive en un contexto social donde el } \\
\text { consumo excesivo de alcohol es esperado y } \\
\text { promovido por parte de amigos y familiares. }\end{array}$ & $\begin{array}{l}\text { 7. Que a las fiestas a las que acudo se acostumbre beber en } \\
\text { exceso. } \\
\text { 8. Que los jóvenes con los que me junto acostumbren beber } \\
\text { en exceso casi todos los fines de semana. } \\
\text { 9. Que los jóvenes con los que me junto consideren normal } \\
\text { beber en exceso. } \\
\text { 10. Que en mi familia se permita beber alcohol en exceso. } \\
\text { 11. Que mis familiares me inviten a beber con ellos. } \\
\text { 12. Que en mi familia se acostumbre beber en exceso } \\
\text { frecuentemente. } \\
\text { 13. Que alguno de mis seres queridos beba muy a menudo. }\end{array}$ \\
\hline $\begin{array}{l}\text { Ausencia de soporte social } \\
\text { por parte de personas } \\
\text { significativas }\end{array}$ & $\begin{array}{l}\text { El sujeto no recibe incentivos sociales para cambiar } \\
\text { su manera de beber, por parte de personas } \\
\text { significativas para él. }\end{array}$ & $\begin{array}{l}\text { 14. Que mis amigos(as) no me motiven a buscar ayuda. } \\
\text { 15. Que mis amigos(as) no apoyen mi decisión de buscar } \\
\text { ayuda. } \\
\text { 16. Que mis padres no me motiven a buscar ayuda. } \\
\text { 17. Que mis padres no apoyen mi decisión de buscar ayuda. }\end{array}$ \\
\hline
\end{tabular}


ANEXO 1. Categorías, definiciones y banco de reactivos obtenidos en los grupos focales y mediante jueces expertos para ambas escalas (continuación)

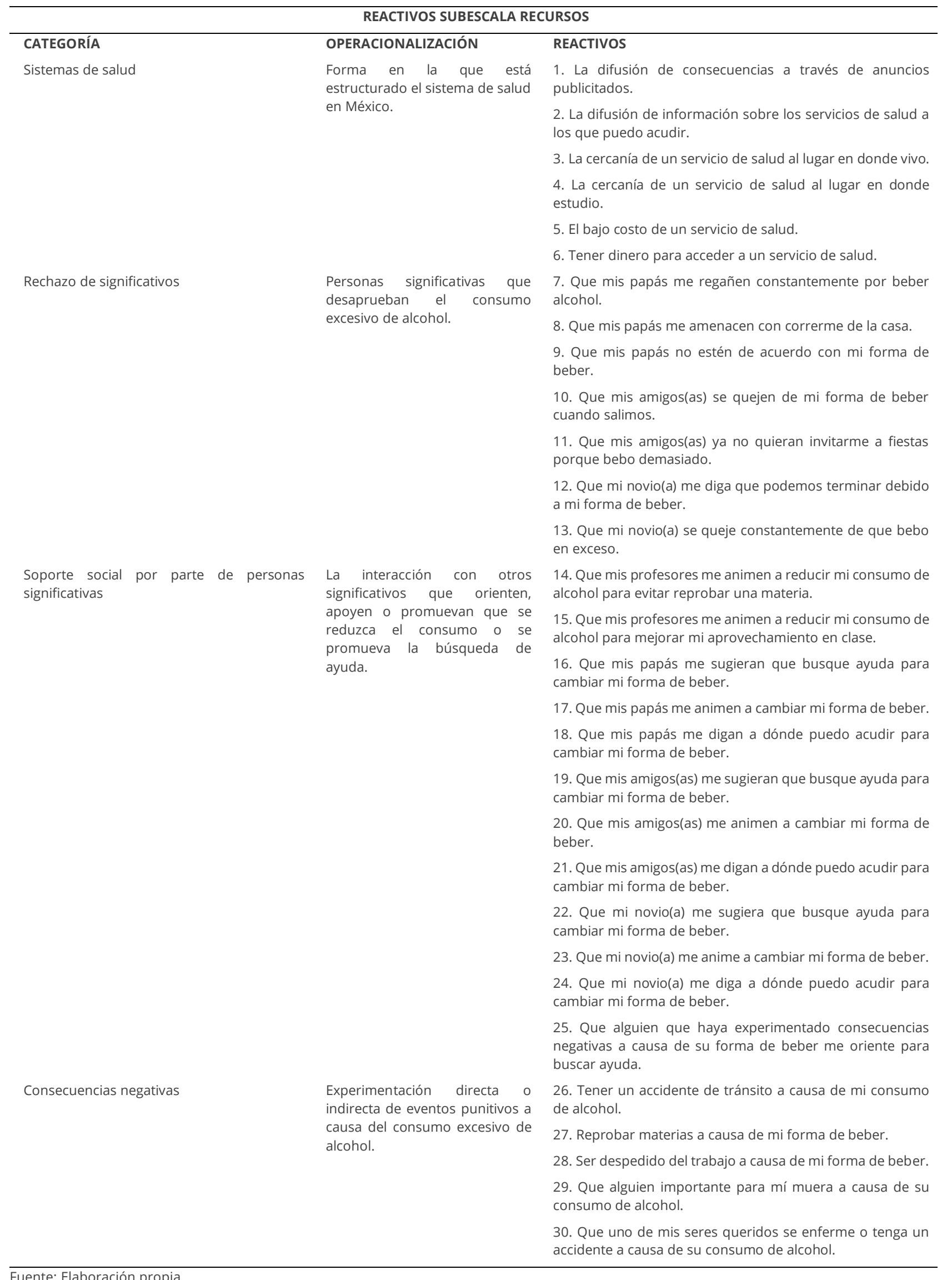

Fuente: Elaboración propia. 
ANEXO 2. Instrumento final. Barreras y Recursos para la Búsqueda de Ayuda en el Cambio del Consumo de Alcohol de Universitarios

INSTRUCCIONES GENERALES: MARCA CON UNA X, QUÉ TAN DE ACUERDO ESTÁS CON LAS SIGUIENTES SITUACIONES.

\begin{tabular}{|c|c|c|c|c|}
\hline SITUACIONES QUE IMPEDIRÍAN QUE BUSCARAS AYUDA... & $\begin{array}{l}\text { Totalmente en } \\
\text { desacuerdo }\end{array}$ & $\begin{array}{l}\text { Poco de } \\
\text { acuerdo }\end{array}$ & $\begin{array}{l}\text { Muy de } \\
\text { acuerdo }\end{array}$ & $\begin{array}{l}\text { Totalmente } \\
\text { de acuerdo }\end{array}$ \\
\hline 1. Que mis padres no apoyen mi decisión de buscar ayuda. & & & & \\
\hline \multicolumn{5}{|l|}{$\begin{array}{l}\text { 2. Que los jóvenes con los que me junto consideren normal beber en } \\
\text { exceso. }\end{array}$} \\
\hline \multicolumn{5}{|l|}{ 3. Que alguno de mis seres queridos beba alcohol muy a menudo. } \\
\hline \multicolumn{5}{|l|}{$\begin{array}{l}\text { 4. Que los jóvenes con los que me junto acostumbren beber en } \\
\text { exceso casi todos los fines de semana. }\end{array}$} \\
\hline \multicolumn{5}{|l|}{$\begin{array}{l}\text { 5. Que mis padres no me motiven a buscar ayuda para cambiar mi } \\
\text { forma de beber alcohol. }\end{array}$} \\
\hline \multicolumn{5}{|l|}{$\begin{array}{l}\text { 6. Que mis amigos(as) no apoyen mi decisión de buscar ayuda para } \\
\text { cambiar mi forma de beber alcohol. }\end{array}$} \\
\hline \multicolumn{5}{|l|}{$\begin{array}{l}\text { 7. Que la gente crea que no puedo disminuir mi consumo de alcohol } \\
\text { por mí mismo(a). }\end{array}$} \\
\hline \multicolumn{5}{|l|}{$\begin{array}{l}\text { 8. Que mis amigos(as) no me motiven a buscar ayuda para cambiar } \\
\text { mi forma de beber alcohol. }\end{array}$} \\
\hline \multicolumn{5}{|l|}{$\begin{array}{l}\text { 9. Que mis amigos(as) ya no me consideren divertido(a) por querer } \\
\text { reducir mi consumo de alcohol. }\end{array}$} \\
\hline SITUACIONES QUE FACILITARÍAN QUE BUSCARAS AYUDA... & $\begin{array}{l}\text { Totalmente en } \\
\text { desacuerdo }\end{array}$ & $\begin{array}{l}\text { Poco de } \\
\text { acuerdo }\end{array}$ & $\begin{array}{l}\text { Muy de } \\
\text { acuerdo }\end{array}$ & $\begin{array}{l}\text { Totalmente } \\
\text { de acuerdo }\end{array}$ \\
\hline \multicolumn{5}{|l|}{$\begin{array}{l}\text { 1. Que mis papás no estén de acuerdo con mi forma de beber } \\
\text { alcohol. }\end{array}$} \\
\hline \multicolumn{5}{|l|}{$\begin{array}{l}\text { 2. Que mis amigos(as) se quejen de mi forma de beber cuando } \\
\text { salimos. }\end{array}$} \\
\hline \multicolumn{5}{|l|}{$\begin{array}{l}\text { 3. Que uno de mis seres queridos se enferme o tenga un accidente a } \\
\text { causa de su forma de beber alcohol. }\end{array}$} \\
\hline \multicolumn{5}{|l|}{$\begin{array}{l}\text { 4. Que mis papás me regañen constantemente por beber alcohol en } \\
\text { exceso. }\end{array}$} \\
\hline \multicolumn{5}{|l|}{$\begin{array}{l}\text { 5. Tener un accidente de tránsito a causa de mi forma de beber } \\
\text { alcohol. }\end{array}$} \\
\hline \multicolumn{5}{|l|}{$\begin{array}{l}\text { 6. Que alguien importante para mí muera a causa de su manera de } \\
\text { beber alcohol. }\end{array}$} \\
\hline \multicolumn{5}{|l|}{$\begin{array}{l}\text { 7. Que mis papás me amenacen con correrme de la casa por mi } \\
\text { forma de beber. }\end{array}$} \\
\hline \multicolumn{5}{|l|}{$\begin{array}{l}\text { 8. Que mi novio(a) se queje constantemente de que bebo alcohol en } \\
\text { exceso. }\end{array}$} \\
\hline 9. Tener dinero para acceder a un servicio de salud. & & & & \\
\hline \multicolumn{5}{|l|}{$\begin{array}{l}\text { 10. Que mi novio(a) me diga que podemos terminar debido a mi } \\
\text { forma de beber alcohol. }\end{array}$} \\
\hline \multicolumn{5}{|l|}{$\begin{array}{l}\text { 11. Que mis amigos(as) me sugieran que busque ayuda para cambiar } \\
\text { mi forma de beber alcohol. }\end{array}$} \\
\hline 12. El bajo costo de un servicio de salud. & & & & \\
\hline
\end{tabular}

